\title{
Mediastinal mass, an extremely rare incidental finding: saccular superior vena cava aneurism
}

\author{
Pedro María Azcarate ${ }^{1 *}$, Jose Antonio Arrieta ${ }^{2}$ Uxua Idiazabal $^{2}$ and Fernando Idoate ${ }^{3}$ \\ ${ }^{1}$ Cardiology Department, Hospital San Pedro. La Rioja, Spain \\ ${ }^{2}$ Cardiology Department, Clinica San Miguel. Pamplona, Spain \\ ${ }^{3}$ Radiology Department, Clinica San Miguel. Pamplona, Spain
}

\begin{abstract}
Aneurysms of the superior vena cava (SVC) are extremely rare. They are usually an incidental finding during the performance of a chest radiography, computed tomography $(\mathrm{CT})$ or magnetic resonance imaging (MRI). The differential diagnosis includes much more frequent pathological findings such as neoplasms, cystic lesion or arterial aneurisms.
\end{abstract}

Since 1949 less than 40 cases of SVC aneurysms have been described in the literature [1]. It appears that most of these aneurisms are fusiform, we report a more unusual saccular aneurism of SVC.

\section{Case}

86 years old patient with hypertension and dyslipemia. In 2004 triple aorto-coronary by-pass was performed. Since 2013 the patient has had 3 admissions for heart failure. The last echocardiogram showed an LVEF of 35\% (poor echocardiographic window). MRI of the heart was requested to assess ventricular volumes and ejection fraction.

MRI most significant findings: dilated left ventricle (end diastolic volume: $393 \mathrm{ml}$ ) with severely depressed systolic function (ejection fraction: 0.28). A spherical mass in medial superior mediastinum (65 x $45 \times 52 \mathrm{~mm}$ ) was found in haste sequences (high signal intensity; Figure 1).

The chest radiography was re-analyze: no mass was found when the patient was in orthostatic position. The mass only shows up when we explored the patient in supine position (Figure 2).

CT findings: saccular superior vena cava aneurysm y right superiormiddle position of $65 \times 45 \times 52 \mathrm{~mm}$ that does not produce compression of the adjacent anatomical structures (Figures 3 and 4 ).

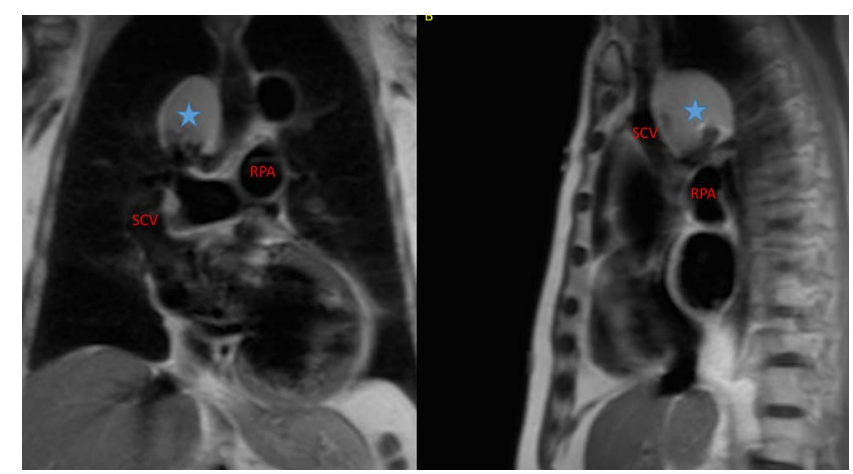

Figure 1. MRI haste sequence; A: coronal view; B: sagittal view; SVC: superior vena cava; RPA: right pulmonary artery; blue star: mediastinal mass.

\section{Discussion}

Mediastinal vein aneurysms are extremely rare. In most cases their cause is unknown [2]; congenital forms, association with cystic higroma and deficiency in the longitudinal muscle layer of the adventicia has also been described [3]. Fusiform aneurysm is the most frequent form. In our case we present a saccular aneurysm, which is even much rarer; this explains why it's only seen when the patient is in supine position. The bright appearance of the aneurysm in the MRI haste ("black blood") sequence is due to blood stasis.

Venous aneurysms are known to develop thrombosis with or without pulmonary embolism or venous obstruction, and may result in rupture [4]. In our case albeit aneurism morphology (saccular) and dimensions ( $65 \times 45 \times 52 \mathrm{~mm}$ ) [5], were enough reasons to send him

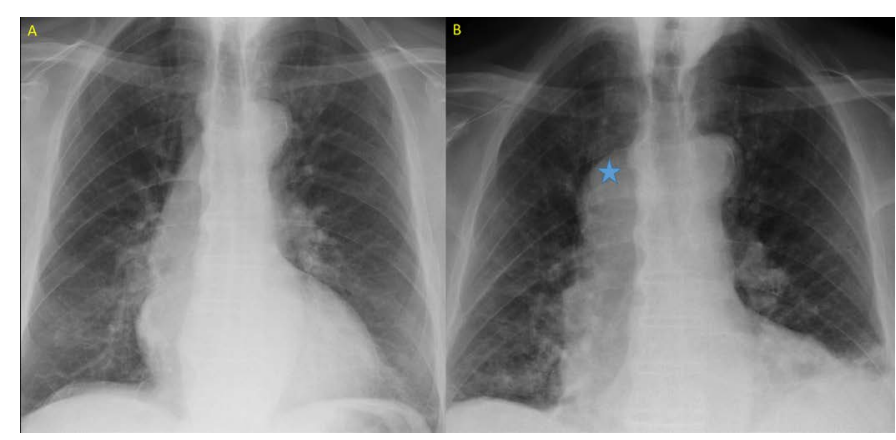

Figure 2. Chest radiography. A: orthostatic position; B: supine position (note there is also interstitial infiltration of the left pulmonary inferior lobe, due to concomitant infectious process). Blue star: mediastinal mass.

Correspondence to: Pedro María Azcarate (Azcarate P.M) MD, PhD, Cardiology Department, Hospital San Pedro, La Rioja, Spain

Received: November 06, 2016; Accepted: November 23, 2016; Published: November 26, 2016 


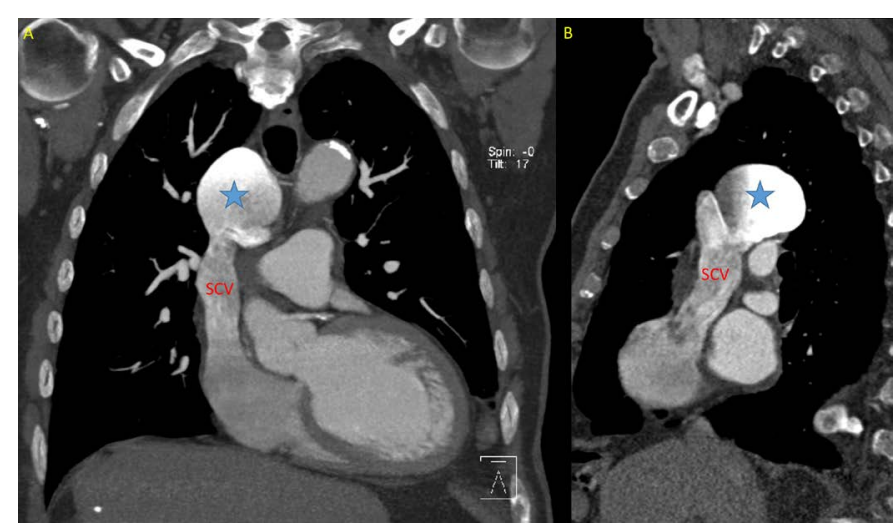

Figure 3. CT A: coronal view; B: sagital view; SVC: superior vena cava; blue star: superio vena cava aneurism

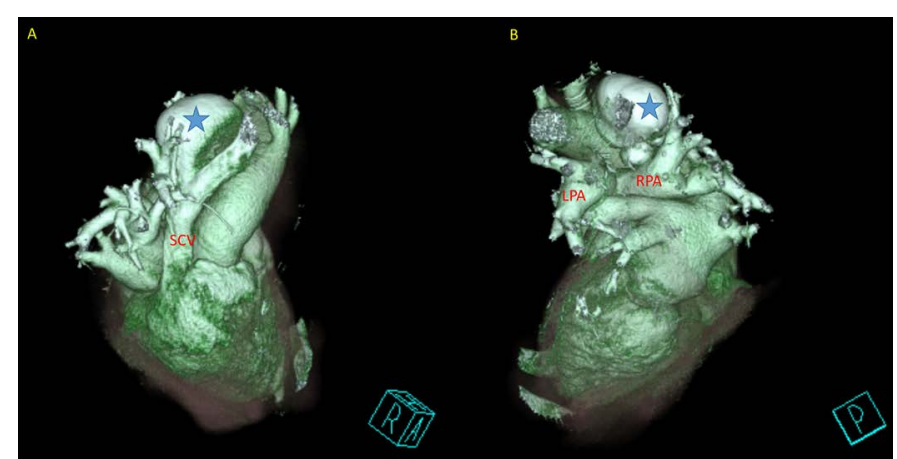

Figure 4. CT Volume rendering; A: anterior view; B: posterior view; SVC: superior vena cava; LPA: left pulmonary artery; RPA: right pulmonary artery; blue star: superior vena cava aneurism. to surgery, due to the patient age and co-morbidities we decided to follow up the patient (he was already under anti-plaque therapy): nonsymptomatic incidental finding [6].

\section{References}

1. Abbott OA, Leigh TF (1964) aneurysmal dilatations of the superior vena caval system. Ann Surg 159: 858-872. [Crossref]

2. Pasic M, Schöpke W, Vogt P, von Segesser L, Schneider J, et al. (1995) Aneurysm of the superior mediastinal veins. J Vasc Surg 21: 505-509. [Crossref]

3. Panduranga P, Thomas E, Al-Maskari S, Al-Farqani A (2012) Giant superior vena caval aneurysm in a post-Glenn patient. Interact Cardiovasc Thorac Surg 14: 878-879. [Crossref]

4. Oh SG, Kim KH, Seon HJ, et al, (2011) Unusual cause of acute right ventricular dysfunction: rapid progression of superior vena cava aneurysm complicated by thrombosis and pulmonary thromboembolism. J Korean Med Sci 26: 690-693.

5. Gabrielli R, Vitale S, Costanzo A, Carra A (2010) Our experience of popliteal vein aneurysm. Interact Cardiovasc Thorac Surg 11: 835-837. [Crossref]

6. Janczak D, Skiba J, Gemel M, Mak M, Ziomek A, et al. (2016) Giant saccular superior vena cava aneurysm-a rare and difficult clinical case. J Thorac Dis 8: E247-249. [Crossref]

Copyright: (C2016 Azcarate PM. This is an open-access article distributed under the terms of the Creative Commons Attribution License, which permits unrestricted use, distribution, and reproduction in any medium, provided the original author and source are credited. 